\title{
Quercetin Enhances Human Sperm Motility in a Dose and Time Dependent Manner
}

\author{
Seda Karabulut $1^{1,2}$, Oya Korkmaz ${ }^{1,2}$, Ceren Erdem Altun ${ }^{1,2}$, Asuman Demiroğlu \\ Zergeroğlü, İlknur Keskin ${ }^{1,2}$ \\ 1 Istanbul Medipol University, International School of Medicine, Histology and Embryology Department, Beykoz, Istanbul, \\ Turkey \\ 2 Istanbul Medipol University, School of Medicine, Kavacık, İstanbul, REMER (Regenerative and Restorative Medicine \\ Research Center) Beykoz, İstanbul, Turkey \\ 3 Gebze Technical University, Department of Molecular Biology and Genetic, Gebze Teknik Üniversitesi Rektörlüğü 41400 \\ Gebze, Kocaeli, Turkey
}

\begin{abstract}
The aim of this study was to investigate the effect of quercetin on the motility of ejaculated human spermatozoa in asthenozoospermic cases by using different doses and exposure times. Semen samples of 94 men were incubated with quercetin at different doses and durations. Sperm motility was analysed in each group, and the results were compared. Compared to control, Quercetin improved sperm motility in each molarity and each interval except $1 \mathrm{M}$. Statistically significant increase was assessed at 0.05 $\mathrm{M}$ after 1 hours of incubation, and $0.1 \mathrm{M}$ after two hours of incubation ( $p<0.05$ ). According to our results, it can be suggested that quercetin has a positive effect on sperm motility on a dose and time dependent manner. This study provides evidence for the potential use of quercetin for sperm preparation to be used in assisted reproduction techniques especially in cases of asthenozoospermia.
\end{abstract}

Keywords: Sperm, motility, quercetin, asthenozoospermia

\section{INTRODUCTION}

Infertility is defined as inability to conceive after one years of unprotected course. Male factor infertility occupies $40 \%$ of the infertility causes and semen quality occupies $70 \%$ of total male infertility reasons. Decreased values of sperm concentration, motility and morphology defined by WHO is known to impair sperm function thus effects fertility. ${ }^{1}$ There is no prov-

\footnotetext{
*Corresponding Author: Seda Karabulut, e-mail: sedakarabulut@medipol.edu.tr Seda Karabulut ORCID Number: 0000-0003-3302-5004 Oya Korkmaz ORCID Number: 0000-0003-2923-5869

Ceren Erdem Altun ORCID Number: 0000-0001-7446-472X

Asuman Demiroğlu Zergeroğlu ORCID Number: 0000-0001-6272-7158

İlknur Keskin ORCID Number: 0000-0002-7059-1884

(Received 27 September 2019, accepted 08 January 2020)
} 
en treatment strategies to overcome the sperm problems. Therefore the couples facing the situation is advised to undergo an assisted reproduction technique including intrauterine insemination (IUI) and intracytoplasmic sperm injection (ICSI). Motility comprises an important problem in both of the techniques especially in IUI cycles where the sperm fertilizes the oocyte spontaneously. There have been numerous studies in the literature regarding sperm motility enhancement strategies whose results are controversial. ${ }^{2}$ Pentoxifyllin is a synthetic dimethylxanthine derivative which is one of the most widely used agent to improve sperm motility ${ }^{3}$ but it is reported to be toxic in longer exposure times. ${ }^{4}$

Flavonoids are polyphenolic compounds that are found in many plantbased foods, including fruits, vegetables, and tea which have been reported to prevent from a wide variety of diseases such as cancer and cardiovascular diseases by acting as an antioxidant. ${ }^{5}$ Amongst the flavonoids, Quercetin is one of the most studied one because of its free radical scavenging and metal chelating abilities. ${ }^{6}$ Quercetin acts as an antioxidant by scavenging ROS and thus suggested to have anticarcinogenic, antiinflammatory and antiviral roles.7 Moreover, Quercetin is suggested to act by protecting against DNA damage ${ }^{8}$ and may be considered as an effective motility enhancement factor for treating male factor infertility.

The effect of quercetin on the sperm cells of several animal species show conflicting results. It was shown that quercetin inhibited rat sperm motility, but a contrary result was obtained with bovine spermatozoa. The aim of this study was to investigate the effect of quercetin on the motility of ejaculated human spermatozoa. We analyzed for the first time the effect of quercetin on sperm motility by using different doses and different exposure times.

\section{METHODOLOGY}

Semen samples of 94 men were obtained from the IVF Center of Medistate Hospital, that applied the clinic because of infertility from August 2018 to January 2019. Patients's semen analysis were performed according to the World Health Organization (WHO) semen analysis guideline. The exclusion criteria were as follows: presence of azoospermia/cryptozoospermia, presence of any kind of chromosomal abnormalities and/or point mutations including AZFy deletions, varicocele, patients with smoking history or alcohol consumption and evidence of infection suggested by the presence of leukocytes on semen analysis. 
Semen samples were analyzed according to WHO criteria. ${ }^{9}$ Sperm samples were collected after 3-7 days of sexual abstinence by masturbation and semen analysis was performed as previously reported. ${ }^{10}$ Shortly, after determining liquefaction time, volume, appearance, $\mathrm{Ph}$ and viscosity of semen samples, sperm concentration $(\mathrm{mil} / \mathrm{mL})$, forwardly progressive sperm motility (A motility) and total motility rates were assessed. At least 100 spermatozoa were scored for motility assessment and motility patterns were classified into four grades as follows: A motility for forward progressive; B motility as, slow non-progressive; C motility as, sluggish and D motility as non-motile motility. Total motility rate was calculated as the sum of $\mathrm{A}, \mathrm{B}$ and $\mathrm{C}$ motility rates.

Sperm samples were divided into 6 aliquots and incubated with different quercetin concentrations of $0,05-0,1-0,2-0,5-$ and $1 \mathrm{M}$ with a final mixture of 1:1 (semen + quercetin) respectively. No quercetin was added to one of the semen aliquots which is classified as the control group. Motility rates of each group were assessed in the first, second and third hours and were compared for each other.

Statistical analyses were performed using Statistical Package for Social Sciences (SPSS, Version 21 for Windows; SPSS, Inc., Chicago, IL, USA). Mann-Whitney-U test were conducted to compare the quantitative variables. The data were expressed as mean percentage. All tests were conducted using a $p$-value $\leq 0.05$ defining statistical significance.

\section{RESULTS AND DISCUSSION}

We observed a time and dose dependent change in motility patterns of each group (Figure 1). Quercetin improved sperm motility in each of the groups and each interval except $1 \mathrm{M}$ when compared with the control but the statistically significant increase was assessed at $0.05 \mathrm{M}$ after 1 hours of incubation and $0.1 \mathrm{M}$ after two hours of incubation ( $\mathrm{p}<0.05$ ). $1 \mathrm{M}$ quercetin showed a toxic effect as assessed by a significant decrease in motility patterns (Figure 1). 


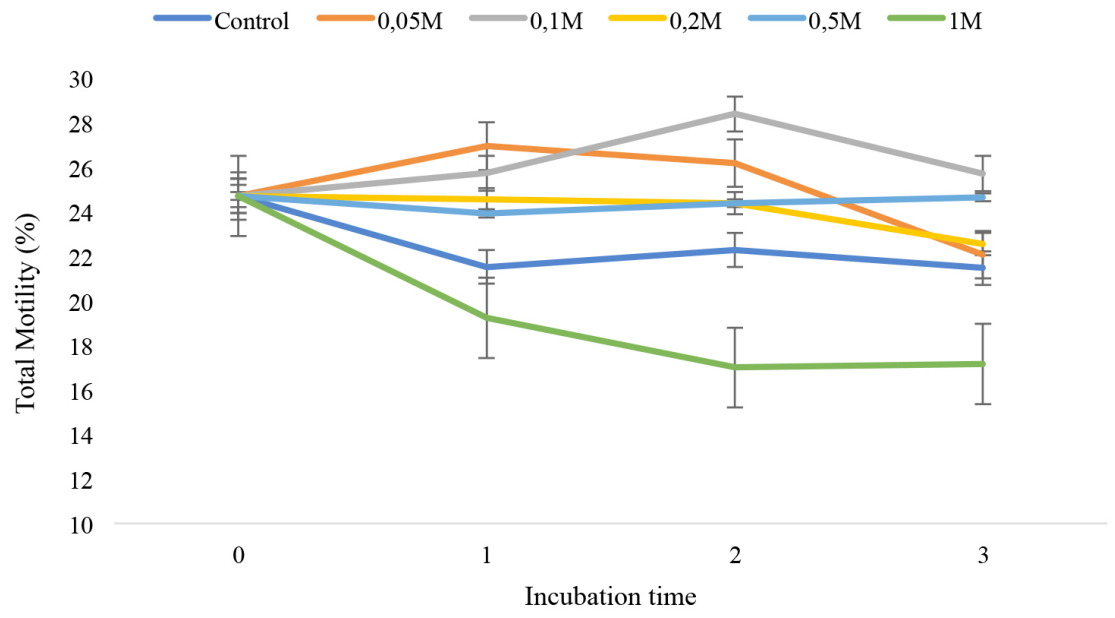

Figure 1. Sperm total motility values after different doses and periods of quercetin exposure

The other semen parameters including sperm concentration, normal morphology and acrosomal index were not changed after the addition of quercetin (Table 1). 


\begin{tabular}{|c|c|c|c|c|c|}
\hline \multirow{4}{*}{$\stackrel{0}{\Sigma}$} & ल) & & $\stackrel{20}{=}$ & & \\
\hline & $\tilde{\sim}$ & & $\simeq$ & & \\
\hline & $\risingdotseq$ & & 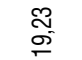 & & \\
\hline & 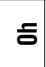 & 吾 & $\begin{array}{l}\text { re } \\
\text { a }\end{array}$ & $\mp$ & 苛 \\
\hline \multirow{4}{*}{ 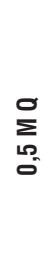 } & $\bar{m}$ & & 㟔 & & \\
\hline & $\overline{\mathbf{N}}$ & & 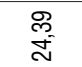 & & \\
\hline & $\mp$ & & $\begin{array}{l}\tilde{\widetilde{N}} \\
\text { స్ }\end{array}$ & & \\
\hline & $\overline{0}$ & $\approx$ & 氐 & $\sigma$ & fี \\
\hline \multirow{4}{*}{$\underset{\substack{0 \\
N}}{\substack{0 \\
0}}$} & $\bar{m}$ & & $\stackrel{\stackrel{\leftrightarrow}{\sim}}{\underset{N}{N}}$ & & \\
\hline & $\overline{\mathbf{N}}$ & & 㞼 & & \\
\hline & $\risingdotseq$ & & 喿 & & \\
\hline & s & $\stackrel{\llcorner}{\sim}$ & 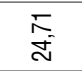 & $\stackrel{\Re}{\forall}$ & 尔 \\
\hline \multirow{4}{*}{ 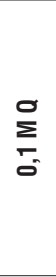 } & $\bar{m}$ & & 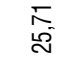 & & \\
\hline & $\overline{\mathrm{N}}$ & & 㤻 & & \\
\hline & $\mp$ & & 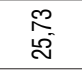 & & \\
\hline & $\overline{0}$ & $\bar{\sim}$ & 䒺 & $\stackrel{\sim}{\sim}$ & F \\
\hline \multirow{4}{*}{$\begin{array}{l}0 \\
\sum_{\mathbf{z}} \\
\stackrel{8}{0} \\
0\end{array}$} & ल & & ๙ิ & & \\
\hline & $\overline{\mathrm{N}}$ & & $\frac{\infty}{c o}$ & & \\
\hline & $\risingdotseq$ & & $\begin{array}{l}\text { co } \\
\text { ci } \\
\text { N }\end{array}$ & & \\
\hline & 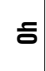 & $\stackrel{2}{\sim}$ & 交 & $\sigma$ & f \\
\hline \multirow{4}{*}{$\begin{array}{l}\text { 흘 } \\
\text { 言 }\end{array}$} & $\bar{m}$ & & $\frac{F}{d}$ & & \\
\hline & $\overline{\text { న }}$ & & \begin{tabular}{l}
$\infty$ \\
\multirow{\sim}{*}{}
\end{tabular} & & \\
\hline & $\mp$ & & $\stackrel{\tilde{N}}{\stackrel{N}{N}}$ & & \\
\hline & $\overline{0}$ & $\stackrel{\sim}{\circ}$ & 吝 & $\widetilde{f}$ & 疋 \\
\hline 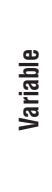 & & 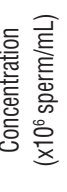 & 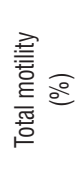 & 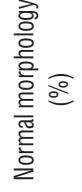 & 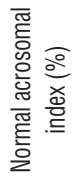 \\
\hline
\end{tabular}


The use of flavonoids for human health as a preventive and/or therapeutic have attracted increasing attention nowadays. Quercetin which is a flavonol-type flavonoid is one of those whose biological effects seem to be associated with its antioxidant role with a wide variety of biological activities, including antibacterial, antiviral, anti-inflammatory, anti-allergic, antiinflammatory, anti-hypertensive, cardio-, neuro-, gastro- hepato-protective and anti-carcinogenic effects ${ }^{11}$ although its cytotoxic effects including apoptosis induction, cell cycle arrest and anti-proliferative effects. Some studies show that quercetin acts as pro-oxidant or antioxidant depending on its concentration. The protective effects of quercetin is suggested to be correlated with the inhibition of lipid peroxidation which is assessed by malondialdehyde (MDA) level measurement. ${ }^{12}$ However, harmful effects of quercetin were also shown which is suggested to be caused by mutagenic and DNA-damaging activities. ${ }^{13}$ Male infertility is responsible for $40 \%$ of all infertility cases in which abnormalities in semen parameters comprise $60 \%$ of them. Motility is one of the most important semen parameters which is accepted to be classified as abnormal below 50\% according to WHO criteria. The studies focusing on sperm motility enhancement mostly focus on antioxidants which have proven to be beneficial in treating several aspects of male infertility. ${ }^{14}$

The effects of quercetin on sperm viability and motility were studied in several animal and human studies with controversial results. Some of these studies concluded that quercetin has a protective or beneficial effect on sperm functions and fertility preservation in several species including

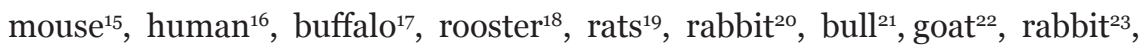
while the others have shown no effect in equine ${ }^{24}$ or negative effect in humans. ${ }^{8}$ Most of these studies have focused on the effect of quercetin as a cryoprotectant during freezing or cold storage. There are only 3 studies that included fresh samples in humans which suggested positive effect in one $^{25}$ and negative effect in two ${ }^{8}$ in which the study of Khanduja et al was the only one that observed motility rates in sperm cells ${ }^{26}$ while the other two analyzed oxidative stress and lipid peroxidation. ${ }^{25}$ This is the second study in the literature that mainly focus on the effect of quercetin on sperm motility rates.

We found a dose and time dependent positive effect of quercetin on sperm motility. Quercetin improved sperm motility in final concentrations of $0,05-0,1-0,2-0,5 \mathrm{M}$, and up to three hours of incubation. Higher concentrations $(1 \mathrm{M})$ were found to have toxic effect and inhibited motility. 
Statistically significant increase was assessed at $0.05 \mathrm{M}$ after 1 hours of incubation and $0.1 \mathrm{M}$ after two hours of incubation. The result obtained in the recent study is not in accordance with the only study of Khanduja et al who reported a dose-dependent fall in sperm motility. The difference obtained may be because of the different concentrations used in this study which is lower than the concentrations used in our study 5-200 $\mu \mathrm{M}$. Moretti et al. (2012) reported that quercetin is effective at low concentrations which has a limited effect on sperm motility and viability but showed to decrease lipid peroxidation ${ }^{25}$ which may partly confirm the findings of our study.

Other studies observing the effect of quercetin on cryopreservation or cold-storage reported its protective and positive effect on post thaw semen parameters including motility, viability, ROS concentration and DNA integrity $^{27}$ which support our results by providing data of frozen sperm samples.

Animal studies including a wide variety of species including buffalo, rooster, rat, rabbit, bull and goat also found a positive effect of quercetin on sperm cells ${ }^{16,18,19,20,21,22,23,24}$ except 2 studies including equine in which they observed no effect ${ }^{24}$ and mice in which it is suggested to induce sperm abnormalities. ${ }^{28}$ Abdallah et al. reported that quercetin may prevent the adverse effects of oxygen radicals, improve the functional parameters of spermatozoa, reduce the levels of lipid peroxidation and increase antioxidant levels in rats. ${ }^{29}$ Quercetin produced a limited positive effect on sperm parameters, but it produced a protective effect by decreasing DNA breaks in sperm cells. However some studies revealed different findings including Jamalan et al.'s study who reported that quercetin do not have a protective effect ${ }^{30}$ against lipid peroxidation induced by metal toxicants; rather, it had inhibitory effects on sperm motility.

According to our results, it can be suggested that quercetin has a positive effect on sperm motility on a dose and time dependent manner. This study provides evidence for the potential use of this flavonoid for sperm preparation to be used in assisted reproduction techniques including Intrauterine insemination (IUI) and Intracytoplasmic Sperm Injection (ICSI) cycles especially in cases of asthenozoospermia.

The findings should be verified by further studies with larger study populations. The molecular mechanisms causing these results and the toxicity assays should be performed before clinic use. 


\section{REFERENCES}

1. WHO laboratory manual for the examination and processing of human semen. World Health Organization Publications, 5th ed.; 2010

2. Wei, C.; Zhang, Y.; Li, R.; Wang, S.; Wang, T.; Liu J.; Liu, Z.; Wang, K.; Liu, J.; Liu, X. Terahertz irradiation-induced motility enhancement and intracellular calcium elevation in human sperm in vitro. Biomed. Opt. Express. 2018, 9, 3998-4008.

3. Banihani, S. A.; Abu-Alhayjaa, R. F. The activity of seminal cre-atine kinase is increased in the presence of pentoxifylline. Andrologia. 2016, 48, 603-604.

4. Sharma, R. K.; Tolentino, M. V.; Jr-Thomas, A. J.; Jr-Agarwal A. Optimal dose and duration of exposure to artificial stimulants in cryopreserved human spermatozoa. J. Urol. 1996, $155,568-573$

5. Ignas, G.; Vilma, P. Relationship between antioxidant and anticancer activity of trihydroxyflavones. Molecules. 2017, 22, 2169.

6. Anand-David, A. V.; Arulmoli, R.; Parasuraman, S.; Overviews of biological importance of quercetin: A bioactive flavonoid. Pharmacogn. Rev. 2016, 10, 84-89.

7. Liang, X. L.; Xia, Z. L.; Yan, J. X. ; Wang, Y. Q.; Xue, S. L.; Zhang, X. H. Quercetin inhibits human sperm functions by reducing sperm $[\mathrm{Ca} 2+] \mathrm{i}$ and tyrosine phosphorylation. Pak. J. Pharm. Sci. 2016, 29, 2391-2396.

8. Chan, S. T.; Lin, Y. C.; Chuang, C. H.; Shiau, R. J.; Liao, J. W.; Yeh, S. L. Oral and intraperitoneal administration of quercetin decreased lymphocyte DNA damage and plasma lipid peroxidation induced by TSA in vivo. Biomed. Res. Int. 2014, 2014, 580626.

9. WHO Laboratory Manual for the Examination of Human Semen and Sperm-Cervical Mucus Interaction. World Health Organization Publications, 4th ed; 1999

10. Yilmaz, S.; Koyuturk, M.; Kilic, G.; Alpak, O.; Aytoz, A. Effects of leucocytospermia on semen parameters and outcomes of intracytoplasmic sperm injection. Int. J. Androl. $\mathbf{2 0 0 5}$, 28, 337-3342.

11. Kawabata, K.; Mukai, R.; Ishisaka, A. Quercetin and related polyphenols: new insights and implications for their bioactivity and bioavailability. Food. Funct. 2015, 6, 1399-1417.

12. Eren-Guzelgun, B.; Ince, E.; Gurer-Orhan, H. In vitro antioxidant/prooxidant effects of combined use of flavonoids. Nat. Prod. Res. 2018, 32, 1446-1450.

13. Johnson, M. K.; Loo, G. Effects of epigallocatechin gallate and quercetin on oxidative damage to cellular DNA. Mutat. Res. 2000, 459, 211-218.

14. Majzoub, A.; Agarwal, A. Antioxidant therapy in idiopathic oligoasthenoteratozoospermia. Indian. J. Urol. 2017, 33, 207-214.

15. Yoshimoto, H.; Takeo, T.; Nakagata, N. Dimethyl sulfoxide and quercetin prolong the survival, motility, and fertility of cold-stored mouse sperm for 10 days. Biol. Reprod. 2017, 97, 883-891.

16. Diao, R.; Gan, H.; Tian, F.; Cai, X.; Zhen, W.; Song, X.; Duan, Y.G. In vitro antioxidation effect of quercetin on sperm function from the infertile patients with leukocytospermia. Am. J. Reprod. Immonul. 2019, 82, 1-7.

17. Ahmed, H.; Jahan, S.; Salman, M. M.; Ullah, F. Stimulating effects of quercetin (QUE) in tris citric acid extender on post thaw quality and in vivo fertility of buffalo (Bubalus bubalis) bull spermatozoa. Theriogenology. 2019, 134, 18-23. 
18. Ghaniel, A.; Eslami, M.; Zadeh-Hashem, E.; Rezapour, R.; Talebi A. Quercetin attenuates $\mathrm{H}_{2} \mathrm{O} 2$-induced toxicity of rooster semen during liquid storage at $4^{\circ} \mathrm{C}$. J. Anim. Physiol. Anim. Nutr. (Berl). 2019, 103, 713-722.

19. Yelumalai, S.; Giribabu, N.; Karim, K.; Omar, S. Z.; Salleh, N. B. In vivo administration of quercetin ameliorates sperm oxidative stress, inflammation, preserves sperm morphology and functions in streptozotocin-nicotinamide induced adult male diabetic rats. Arch. Med. Sci. 2019, 15, 240-249.

20. Naseer, Z.; Ahmad, E.; Şahiner, H. S.; Epikman, E. T.; Fiaz, M.; Yousuf, M. R.; Khan, S. A.; Serin, İ.; Ceylan, A.; Aksoy, M. Dietary quercetin maintains the semen quality in rabbits under sumer heat stress. Theriogenology. 2018, 122, 88-93.

21. Avdatek, F.; Yeni, D.; İnanç, M. E.; Çil, B.; Tuncer, B. P.; Türkmen, R.; Taşdemir, U. Supplementation of quercetin for advanced DNA integrity in bull semen cryopreservation. Andrologia. 2018, $50,1-7$.

22. Seifi-Jamadi, A.; Ahmad, E.; Ansari, M.; Kohram, H. Antioxidant effect of quercetin in an extender containing DMA or glycerol on freezing capacity of goat semen. Cryobiology. 2017, $75,15-20$.

23. Johinke, D.; de Graaf, S. P.; Bathgate, R. Quercetin ruduces the in vitro production of H2O2 during chilled storage of rabbit spermatozoa. Anim. Reprod. Sci. 2014, 151, 208-219.

24. Filho, J. S.; Corcini, C. D.; Santos, F. C. C.; Anciuti, A. N.; Gatti, N. L .S.; Anastacio, E.; Mielke, R.; CEW, N.; Curcio, B. R.; Varela-Junior, A. S. Quercetin in equine frozen sperm. Cryo Letters. 2017, 38, 299-304.

25. Moretti, E.; Mazzi, L.; Terzuoli, G.; Bonechi. C.; Iacoponi, F.; Martini, S.; Rossi, C.; Collodel, G. Effect of quercetin, rutin, naringenin and epicatechin on lipid peroxidation induced in human sperm. Reprod. Toxicol. 2012, 34, 651-657.

26. Khanduja, K. L.; Verma, A.; Bhardwaj, A. Impairment of human sperm motility and viability bu quercetin is independent of lipid peroxidation. Andrologia. 2001, 33, 277-281.

27. Azadi, L.; Tavalaee, M.; Deemeh, M. R.; Arbabian, M.; Nasr-Esfahani, M. H. Effects of tempol and quercetin on human sperm function after cryopreservation. Cryo Letters. 2017, 38, 29-36.

28. Rastogi, P. B.; Levin, R. E. Induction of sperm abnormalities in mice by quercetin. Environ. Mutagen. $1987,9,79-86$.

29. Ben-Abdallah, F.; Zribi, N.; Ammar-Keskes, L. Antioxidative potential of quercetin against hydrogen peroxide induced oxidative stress in spermatozoa in vitro. Andrologia. 2011, 43, 261-265.

30. Jamalan, M.; Ghaffari, M. A.; Hoseinzadeh, P.; Hashemitabar, M.; Zeinali, M. Human Sperm Quality and Metal Toxicants: Protective Effects of some Flavonoids on Male Reproductive Function. Int. J. Fertil. Steril. 2016, 10, 215-223. 